\title{
DEPOLARIZATION LIDAR DETERMINATION OF CLOUD-BASE MICROPHYSICAL PROPERTIES
}

\author{
D.P. Donovan ${ }^{1}$, H. Klein Baltink ${ }^{1}$, J.S. Henzing ${ }^{2}$, S. de Roode ${ }^{3}$ and A.P. Siebesma ${ }^{1,3}$ \\ 1 Royal Netherlands Meteorological Institute (KNMI). PO Box 201, 3730 AE De Bilt, The Netherlands. \\ donovan@knmi.nl \\ 2 Netherlands Organisation for Applied Scientific Research (TNO), Utrecht, The Netherlands. \\ 3 Technical University of Delft (TUD), Delft, The Netherlands.
}

\begin{abstract}
The links between multiple-scattering induced depolarization and cloud microphysical properties (e.g. cloud particle number density, effective radius, water content) have long been recognised. Previous efforts to use depolarization information in a quantitative manner to retrieve cloud microphysical cloud properties have also been undertaken but with limited scope and, arguably, success. In this work we present a retrieval procedure applicable to liquid stratus clouds with (quasi-)linear LWC profiles and (quasi-)constant number density profiles in the cloud-base region. This set of assumptions allows us to employ a fast and robust inversion procedure based on a lookup-table approach applied to extensive lidar Monte-Carlo multiple-scattering calculations. An example validation case is presented where the results of the inversion procedure are compared with simultaneous cloud radar observations. In non-drizzling conditions it was found, in general, that the lidar- only inversion results can be used to predict the radar reflectivity within the radar calibration uncertainty (2-3 dBZ). Results of a comparison between ground-based aerosol number concentration and lidar-derived cloud base number considerations are also presented. The observed relationship between the two quantities is seen to be consistent with the results of previous studies based on aircraft-based in situ measurements.
\end{abstract}

\section{INTRODUCTION}

The fact that a linear polarization lidar will detect a cross-polarized signal due to the occurrence of multiple-scattering in liquid water clouds has been recognised since at least 1970 [1]. Extensive field and laboratory observations [2] of the depolarization of laser radiation in water clouds have been made and various theoretical approaches have been developed ranging from Monte Carlo-based (MC-based) models to semianalytic approaches; see [4] for a review.

In spite of the long history and the increasing understanding of the relevant phenomenon, the use of depolarization measurements to retrieve cloud extinction and microphysical information appears to not have seen widespread implementation. This may be due to the fact that much of the theoretical work has either used homogeneous clouds (over simplified) or on the general problem of retrieving arbitrary cloud property profiles (too ambitious). Yet another reason may be the shift in attention to multi-fieldof-view (FOV) lidars.

\section{METHODOLOGY}

In this work we present a retrieval procedure using single FOV depolarization lidars. The retrieval is based on assuming that the cloud-base region can be characterised by a quasi-linear (with height) LWC profile (i.e. constant LWC lapse rate) and constant cloud particle number density. This set of assumptions allows us to reduce the cloud variables to two parameters. In turn, this enables the development of a fast and robust inversion procedure using a look-up-table approach based on stored results from accurate lidar Monte-Carlo (MC) simulations.

The procedure does not rely on the absolute calibration of the lidar signals. However, the lidar depolarization calibration including cross-talk effects must be accounted for. In order to take into account any errors in the depolarization and crosstalk correction procedures the relevant instrument parameters taken into account using an Optimal 


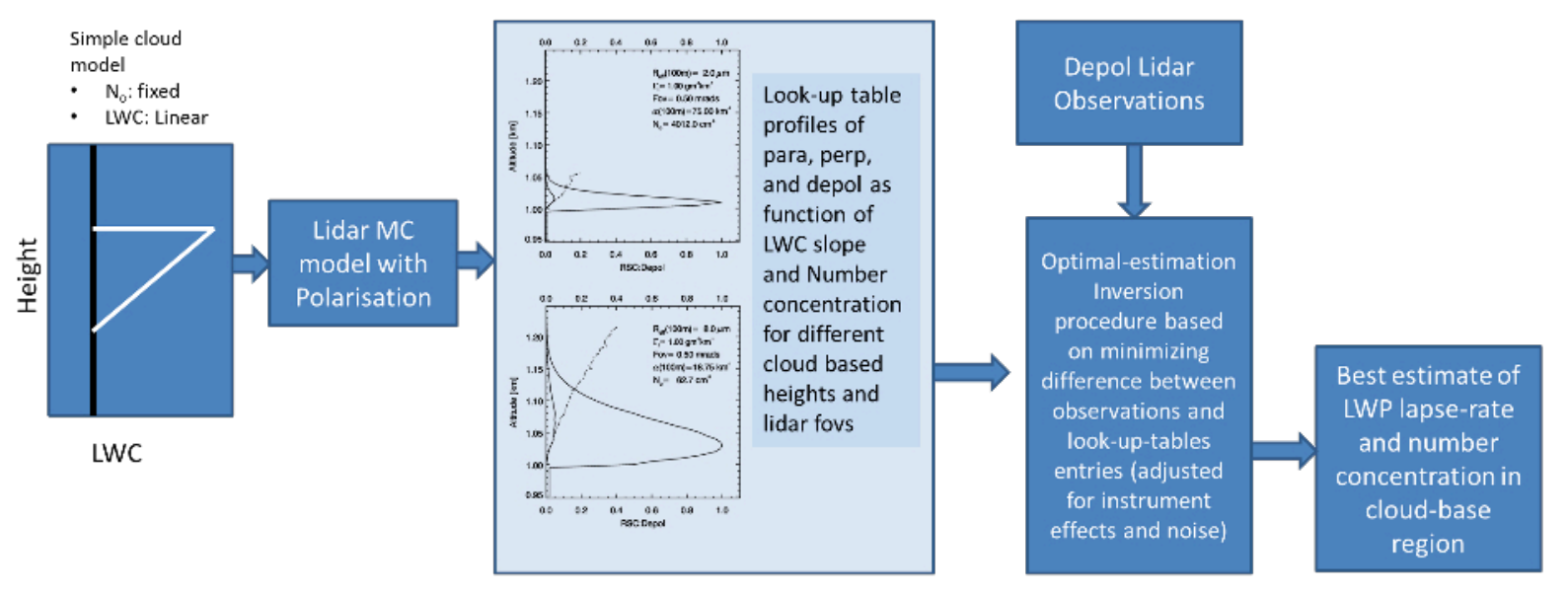

Figure 1: Schematic depiction of the inversion process.

Estimation (OE) approach. This is depicted in Fig.1. Extensive details may be found in [2].

Development and testing of the inversion procedure was facilitated by end-to-end testing of the procedure using Large-Eddy-Simulation
(LES) model data. An example is presented in Fig.2. Here, data from the DALES model [6] driven by observed conditions over the Dutch CESAR measurement site at Cabauw in the central Netherlands were used. The simulations

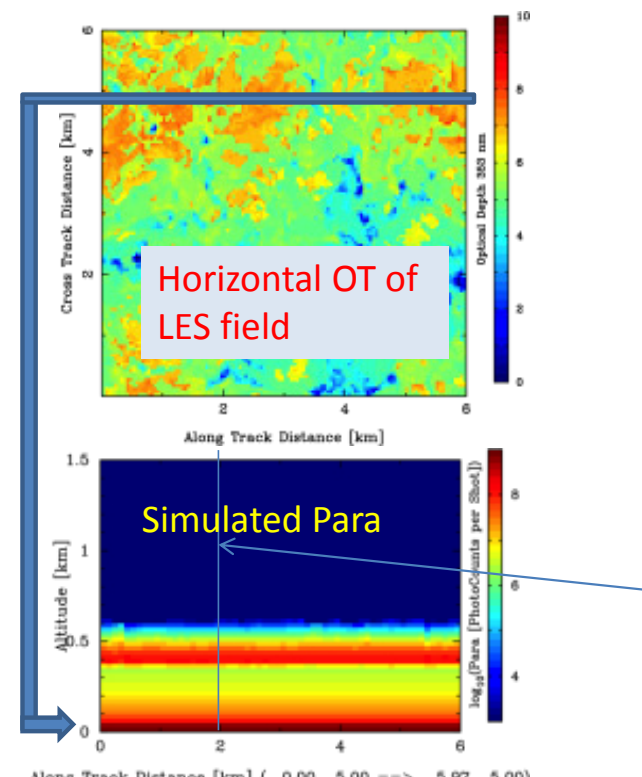

Along Track Distance $[\mathrm{km}](0.00,5.00=->5.97,5.00)$

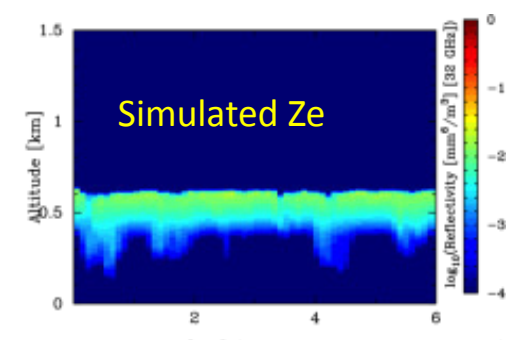

Along Track Distance $[\mathrm{km}](0.05,5.00=->5.96,5.00)$

\section{Simulation Example I}

\section{Inversion approach tested and developed using LES based simulations.}

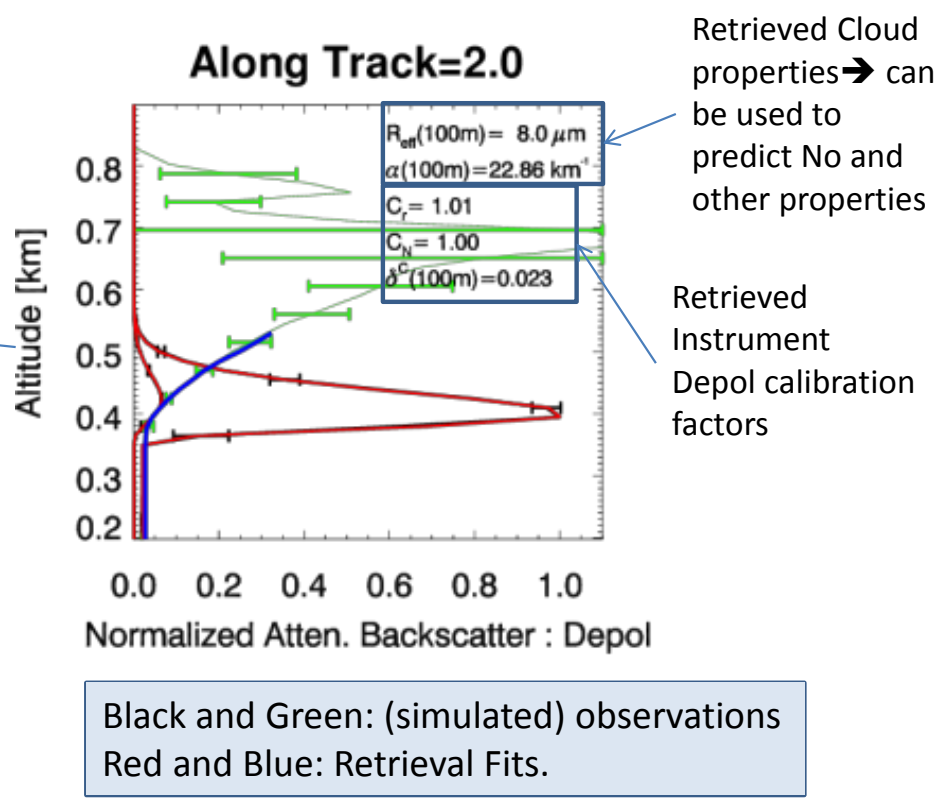

Procedure is "blind" to low levels of drizzle.

Figure 2: Example end-to-end simulation results (for one profile) using LES data. 
indicate that the procedure should produce robust results in the presence of non-ideal cloud macrostructure and instrument noise.

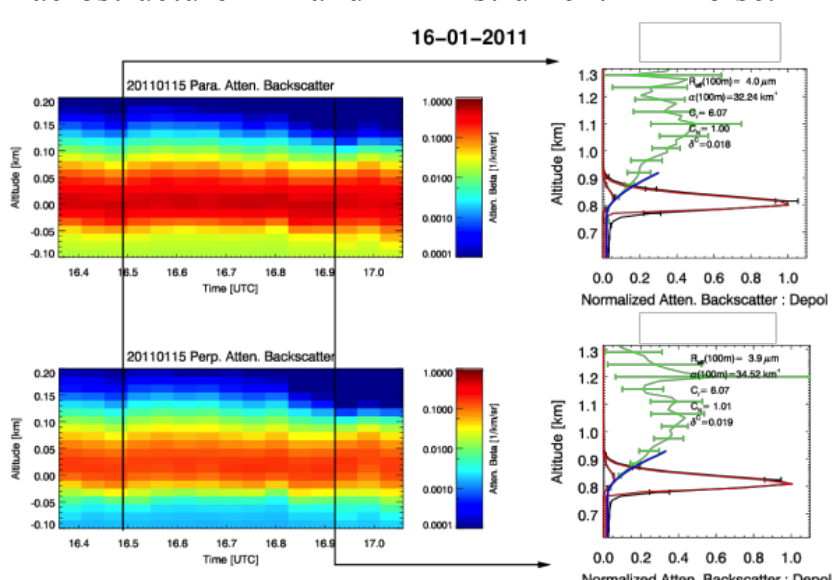

Figure 3: Results of the retrieval applied to lidar data for two periods on 16/01/2011. The Black-lines correspond to the normalized para and perp attenuated backscatters while the observed depol. ratio is given by the Green-line The Red-lines are the fits to the observed attenuated backscatters and the Blue-line is the corresponding fit depolarization ratio. $\Gamma_{l}$ is the liquid water lapse rate, while $\mathrm{Cr}, \mathrm{C}$

and $\delta^{c}$ are instrument parameters associated with the polarization calibration and cross-talk.
It is also interesting to note that, unlike, techniques involving the quantitate use of radar reflectivity that the procedure is robust against the presence of drizzle.

\section{RESULTS}

An example application of the depolarization lidar inversion to real observations is shown in Fig. 3. The lidar observations were made with a $355 \mathrm{~nm}$ Leosphere ALS-450 backscatter lidar. The system has been in operation at Cabauw since mid-2007 with breaks in the record ranging from weeks to several months. The data was acquired with a vertical resolution of $15 \mathrm{~m}$ and a temporal resolution of about $30 \mathrm{~s}$. Here it can be seen that very good fits to actual data are obtained.

As well as the lidar measurements, we also make use of the $35 \mathrm{GHz}$ lidar observations at Cabauw. The cloud radar is a vertically pointing Doppler radar with a vertical resolution of $89 \mathrm{~m}$ and a temporal resolution of approximately $15 \mathrm{~s}$. In Fig.4, time series of lidar-derived parameters for several hours on Jan 15, 2011 are shown. A comparison between the 'cloud-base' radar reflectivity predicted on the basis of the lidar retrieval results and the observed values is also presented. The agreement between the lidar

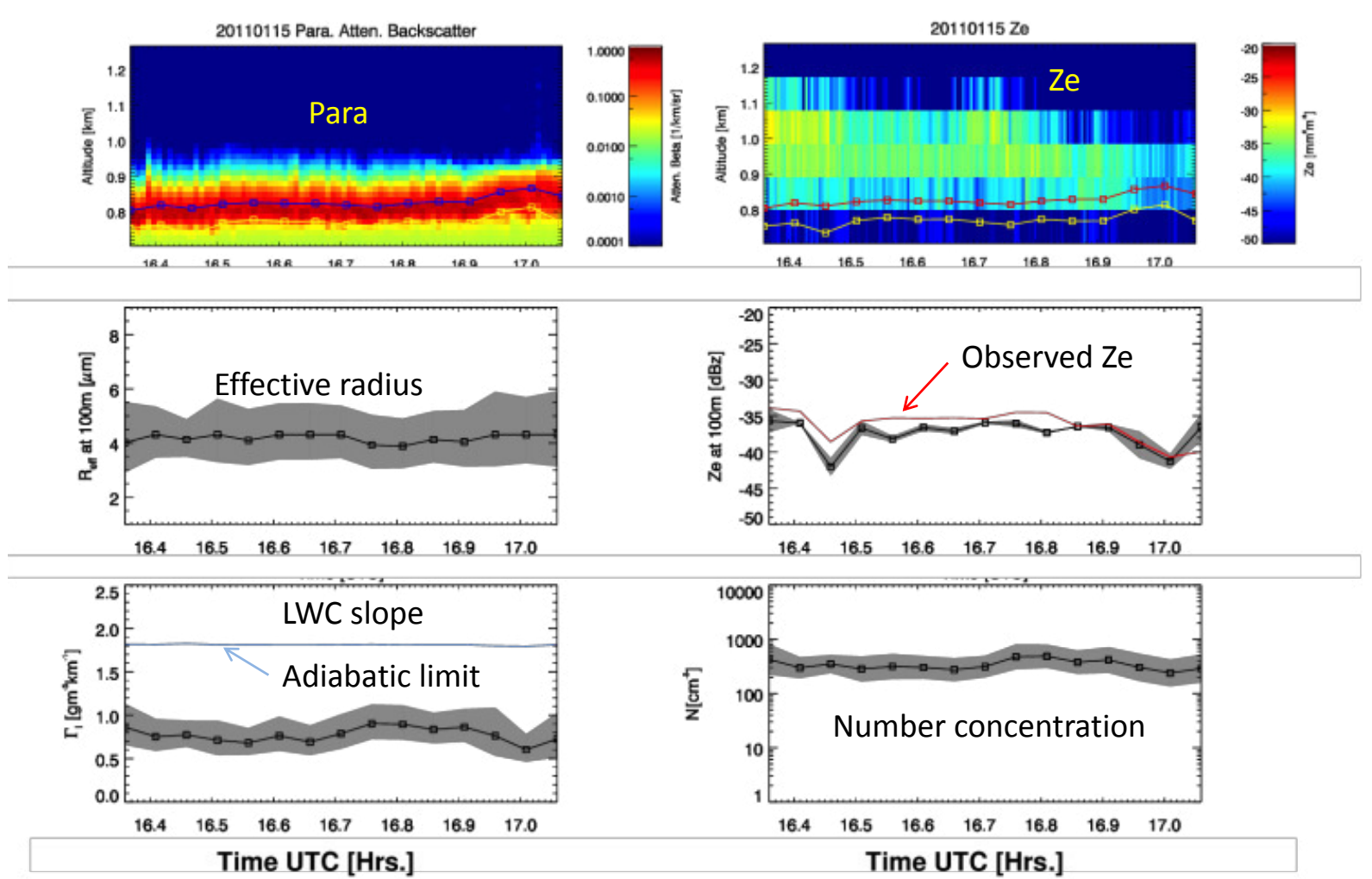

Figure 4: Example time series inversion results including observed radar reflectivities averaged $100 \mathrm{~m}$ above cloud base and reflectivities calculated on the basis of the lidar inversion results. 
derived estimates and the observations agree within the $2-3 \mathrm{dBz}$ calibration uncertainty of the radar. This was generally observed to be the case in non-drizzling conditions and strongly supports the validity of the lidar inversion results.

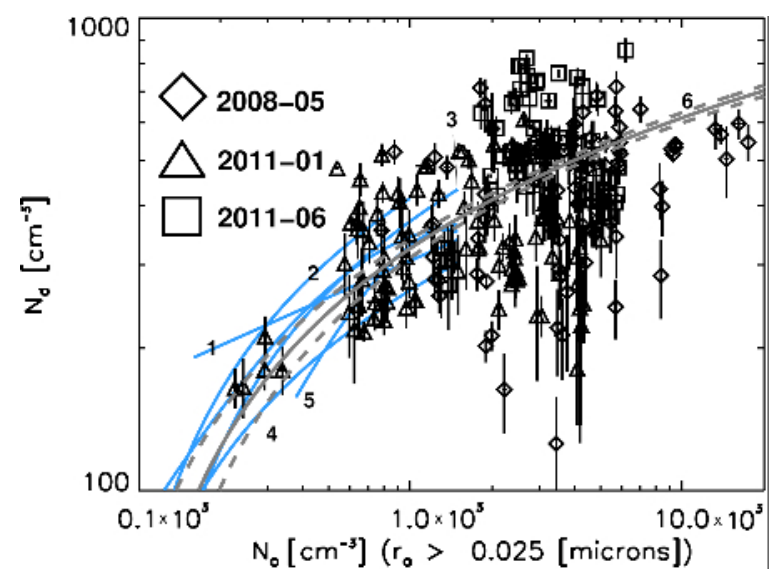

Figure 5: Lidar-derived cloud-base number density (Nd) and SMPS ground-based number density of (Na). The Light-Blue lines represent previous empirical relationships based on in-situ aircraft measurements. Each point represents a $30 \mathrm{~min}$. average result. The data spans about 3 months of observations of boundary layer stratus clouds.

As well as the remote-sensing equipment, Cabauw also hosts a number of in situ probes including a Scanning Mobility Particle Sizer (SMPS) instrument which measures aerosol size distributions between diameters of 10 and $470 \mathrm{~nm}$. As described in [7], the SMPS instrument is connected to a laminar flow sampling tube with an inlet at $60 \mathrm{~m}$.

A comparison between lidar derived cloud number density and in-situ results are shown in Fig. 5. Here a range of boundary-layer cases was considered. It can be seen that the comparison is consistent with a range of previously derived empirical relationships based on in-situ aircraft observations e.g. [8].

\section{CONCLUSIONS}

A new method for retrieving liquid stratus cloudbased microphysical properties using common depolarization lidar signals has been developed. The method appears to produce accurate robust results. This conclusion is based on simulations driven by LES data, consistency with radar observations in non-drizzling clouds and consistency with the results of previous aircraft in-situ based investigations into the correlation between cloud and aerosol number densities. For more information see [2]

\section{ACKNOWLEDGEMENTS}

The aerosol size distribution measurements used in this paper were partly funded by European Union Seventh Framework Program (FP7/20072013) under grant agreement no. 262254.The LES simulations used in this work were sponsored by the Dutch National Computing Facilities Foundation (NCF) who provided the use of supercomputer facilities.

\section{REFERENCES}

[1] Liou, K.-N. and Schotland, R. M., 1971: Multiple backscattering and depolarization from water clouds for a pulsed lidar system, J. Atmos. Sci., 28,772-784.

[2] Sassen, K., 2005: Polarization in lidar, in: Lidar, edited by: Weitkamp, C., vol. 102 of Springer Series in Optical Sciences, 19-42, Springer, New York, doi:10.1007/0-387-251014_2, 2005.

[3] Chaikovskaya, L., 2008: Remote sensing of clouds using linearly and circularly polarized laser beams: techniques to compute signal polarization, in: Light Scattering Reviews 3, edited by: Kokhanovsky, A. A., Springer Praxis Books, 191228, Springer, Berlin Heidelberg.

[5] Donovan, D.P. et al., 2015: A depolarization lidar-based method for the determination of liquid-cloud microphysical properties, Atmos. Meas. Tech., 8, 237-266, 2015 doi:10.5194/amt-8237-2015

[6] Heus, T., van Heerwaarden, et al., 2010: Formulation of the Dutch Atmospheric LargeEddy Simulation (DALES) and overview of its applications, Geosci. Model Dev., 3, 415-444.

[7] Mensah, A. A. et al., 2012: Aerosol chemical composition at Cabauw, The Netherlands as observed in two intensive periods in May 2008 and March 2009, Atmos. Chem. Phys., 12, 4723 4742,doi:10.5194/acp-12-4723-2012.

[8] Leaitch, W. R., et al., 1992: The relationship between cloud droplet number concentrations and anthropogenic pollution: observations and climatic implications, J. Geophys. Res., 97, 2463- 
2474, doi:10.1029/91JD02739. 